\title{
Evolutionary game analysis of enterprise R\&D decision-making in the production and learning alliance
}

\author{
WangYan ${ }^{1, a}$ * \\ ${ }^{1}$ Dalian University of Science And Technology, ${ }^{2}$ Dongbei University of Finance and Economics, \\ Dalian 116052
}

\begin{abstract}
Key words: alliance of academy-industry; Evolutionary game; Cooperative strategy
\end{abstract}
\begin{abstract}
In this paper, the evolution game model is used to investigate the difference of the profit and game strategies of the cooperative innovation of enterprises participating in the cooperative innovation of enterprises under the constraints of enterprise cooperation and non-cooperation (competition). Results indicate that Under the constraint of inter-enterprise cooperative strategy, there are two steady-state convergent equilibrium points. The direction of strategy convergence depends on the net gain of cooperative innovation. Under the constraint of non-cooperative strategy between enterprises, whether the enterprise chooses to participate or not participate fully depends on whether the net income can be obtained under the participation strategy. At the same time, both the increase of gain and the decrease of cost can increase the probability of participation strategy selection. Further, the above analysis shows that the government's policy of stimulating enterprises to participate in the collaborative innovation of production through direct and indirect financial subsidies is reasonable. However, the incentive effect of government subsidy under non-cooperative constraint can realize the existence threshold.
\end{abstract}

\section{Introduction}

In both practical and academic aspects, the positive promotion effect of the alliance on technological innovation has become the consensus. As Henry has said, the synergy between production and learning has shown remarkable innovation performance. Because of the open interaction structure in cooperation, it can accelerate the transfer and sharing of knowledge among the innovation subjects ${ }^{[1]}$. Furthermore, it stimulates the reinvention of dispersed and heterogeneous knowledge, and promotes the benign interaction of the innovation subjects to maximize the knowledge utility but Specific to the inside of production union. A ffirming produce - study in academic circles' cooperation innovation action at the same time, in the same alliance is cooperation or under the competitive pattern among enterprises build stand-alone production better technological innovation synergy innovation body but there is a larger dispute.

Part of the study think that the same production alliance platform cooperative innovation between enterprises have a higher expected return and innovation efficiency.

For example,Groger thinks that due to the increasing complexity of technology, the development uncertainty and development cost will be enhanced. Only collaborative innovation can improve the efficiency of resource allocation and enhance the predictability of innovation ${ }^{[2] .}$

Hitt argues that even if the technology complexity is not high, considering the cooperation of the existence of the technology spillover effect and knowledge learning ability enhancement, collaborative innovation platform in cooperation than competition between enterprises more innovative efficiency ${ }^{[3]}$.

Lichtenthaler also supports this view based on the analysis of the two-stage Cournont game model. It believes that maintaining cooperative relationship between enterprises is more conducive to controlling $\mathrm{r} \& \mathrm{~d}$ cost, and cooperation may also lead to the increase of innovation market income ${ }^{[4]}$. The result of the analysis of the evolutionary game model by suxianna et al. also believes that the net investment of enterprise cooperative innovation is lower and the collaborative efficiency ${ }^{[5]}$.

But other studies have found it more efficient to maintain a competitive relationship while participating in a production-learning alliance. Fisher compares the strategic differences between enterprise cooperation and competition in a group evolution game framework. The conclusion is 
that the technological innovation of emerging industries is more suitable for the construction of a single innovation structure. The conclusion is that the technological innovation of emerging industries is more suitable for the construction of a single innovation structure. Based on the analysis of the three-stage Cournont model, lei jing also believes that based on the production constraints, the construction of competitive production-learning cooperative research and development platforms will be more able to achieve the welfare maximization objective ${ }^{[7]}$.

In addition, Nelson also think enterprise cooperation must be based on an innovative usufruct can clearly defined under the premise of high overflow when innovation, market position is different between enterprises and innovation property right definition is not clear, even if the cooperation is the most conducive to innovation, but it is not the optimal strategy of the company to choose ${ }^{[8]}$. HeJunli also believes that China's cooperative innovation is not guaranteed by the effective system of usufruct. The collaborative innovation of competitive production by enterprises has led to the increase of total social cost, but it is the rational choice of enterprises ${ }^{[9]}$.

Based on the above differences, this paper focuses on the game process of intra-enterprise cooperation and competition strategy. Specifically, this paper USES the evolutionary game model respectively to investigate enterprise cooperative and non-cooperative (competition) constraints, The enterprise participates in the production learning alliance to make the profit level change and the corresponding decision-making process.

Contribution of this paper is mainly reflected in: First, the influence of cooperation and non-cooperation on the decision-making of collaborative innovation is investigated. Second, based on the evolutionary game framework, the influence of synergetic innovation gain and collaborative cost on enterprise participation decision is analyzed.

Competition and cooperation: the enterprise participates in the strategic analysis of the innovation of the alliance.

Enterprises are uncertain and bounded rationality in selecting cooperative innovation strategy, it's a dynamic game over time process ${ }^{[10]}$. To simplify subsequent analysis. Suppose that there are only two companies A and B participating in a new product technology innovation.

The total revenue of the market of technological innovation is $r$, the cost of $r \& d$ input needed for innovation is i. There is no market status difference between enterprises so that the technology innovation dividend can be Shared. At the same time, we have developed a technology cooperative research and development platform for the new technology.

Corporate $r \& d$ decisions include the following options:

H1: Not participating - not participating in the strategy. Enterprises do not participate in the cooperative research and development platform. At this time, enterprise A and B are both closed $r \& d$. $R \& d$ revenue $r$ sharing, At the same time, we need to invest in $r \& d$ cost separately.

H2: Participation - not participating in the strategy. Suppose that one party is involved in the production alliance. But the other party doesn't chooses to participate in the closed development,otherwise participate in the enterprise increase revenue $\triangle \mathrm{r}$, However, it is necessary to bear the additional cost of building the alliance platform, not to participate in the company's earnings reduction.

H3: Participation - non-cooperative strategy. Enterprise A and B choose to build the alliance of obstetrics with the research and development institutions independently and To maintain non-cooperative competitive relations among enterprises. At this time, $R \& d$ revenue $r$ is shared, R\&d initial input cost I remains unchanged, but each bears the additional cost of input.

H4: Participation - cooperation strategy. Enterprise A and B participate in the technical innovation of the alliance platform for production learning. Enterprise A and B participate in the technical innovation of the alliance platform for production learning. cooperative relations is maintained among enterprises. 


\section{Game analysis of enterprise r\&d decision-making under the strategy of inter-enterprise cooperation.}

If two enterprises undertake technical innovation, and satisfy the hypothesis H1, H4. The game return matrix is shown in table 1.

Tab. 1 payoff matrix of cooperative innovation

\begin{tabular}{|c|c|c|}
\hline & participation & nonparticipation \\
\hline participation & $\frac{1}{2}(r-i-\Delta i) \quad \frac{1}{2}(r-i-\Delta i)$ & $\frac{1}{2} r-i+\Delta r-\Delta i \quad \frac{1}{2} r-i-\Delta r$ \\
\hline nonparticipation & $\frac{1}{2} r-i-\Delta r \quad \frac{1}{2} r-i+\Delta r-\Delta i$ & $\frac{1}{2} r-i \quad \frac{1}{2} r-i$ \\
\hline
\end{tabular}

The strategy probability of setting enterprise A to participate is ${ }^{x}$,the strategy probability of not to participate is $1-x$

For enterprise A, data from table 1 can be obtained:

$$
\begin{aligned}
& u_{1 y}=\frac{1}{2}(\mathrm{r}-i-\Delta i) y+\left(\frac{1}{2} \mathrm{r}-i+\Delta r-\Delta i\right)(1-y) \quad u_{1 n}=\left(\frac{1}{2} \mathrm{r}-i-\Delta r\right) y+\left(\frac{1}{2} \mathrm{r}-i\right)(1-y) \\
& \bar{u}_{1}=u_{1 y} x+u_{1 n}(1-x) \quad u_{1 y}-\bar{u}_{1}=(1-x)\left[\left(\frac{1}{2} i+\Delta i\right) y+(\Delta r-\Delta i)\right]
\end{aligned}
$$

Therefore, the replication dynamic equation of enterprise $\mathrm{A}$ is:

$$
\frac{d x}{d t}=x\left(u_{1 y}-\bar{u}_{1}\right)=x(1-x)\left[\left(\frac{1}{2} i+\Delta i\right) y+(\Delta r-\Delta i)\right]
$$

Similarly, for enterprise $\mathrm{B}$, the expected return is:

$$
\begin{gathered}
u_{2 y}=\frac{1}{2}(\mathrm{r}-i) x+\left(\frac{1}{2} \mathrm{r}-i+\Delta r-\Delta i\right)(1-x) \quad u_{2 n}=\left(\frac{1}{2} \mathrm{r}-i-\Delta r\right) x+\left(\frac{1}{2} \mathrm{r}-i\right)(1-x) \\
\bar{u}_{2}=u_{2 y} y+u_{1 n}(1-y) \quad u_{2 y}-\bar{u}_{2}=(1-y)\left[\left(\frac{1}{2} i+\Delta i\right) x+(\Delta r-\Delta i)\right]
\end{gathered}
$$

Therefore, the dynamic equation of enterprise $\mathrm{B}$ is:

$$
\frac{d y}{d t}=y\left(u_{2 y}-\bar{u}_{2}\right)=y(1-y)\left[\left(\frac{1}{2} i+\Delta i\right) x+(\Delta r-\Delta i)\right] \quad \text { let } \frac{d x}{d t}=\frac{d y}{d t}=0
$$

in plane $M=\{(x, y) \mid 0 \leq x \leq 1,0 \leq y \leq 1\}$, the five equilibrium points of this evolutionary game

$$
\text { are: }(0,0),(0,1),(1,0),(1,1) \text { as well as }\left(x^{*}=\frac{2(\Delta i-\Delta r)}{i+2 \Delta i}, y^{*}=\frac{2(\Delta i-\Delta r)}{i+2 \Delta i}\right)
$$

According to the research of Friedman, the stability of the equilibrium point of the evolution system can be obtained by the local stability analysis of the jacobian matrix.

By (1) and (2), the jacobian matrix $\mathrm{J}$ is obtained:

$$
\left[\begin{array}{ll}
(1-2 x)\left[\left(\frac{1}{2}+\Delta i\right) y+(\Delta r-\Delta i)\right] & \frac{1}{2} x(1-x)(\mathrm{i}+2 \Delta \mathrm{i}) \\
\frac{1}{2} y(1-y)(i+2 \Delta i) & (1-2 y)\left[\left(\frac{1}{2}+\Delta i\right) x+(\Delta r-\Delta i)\right]
\end{array}\right]
$$

By computing its jacobian matrix, its determinant and trace are shown in table 2. 
Tab. 2 the result of evolutionary stability

\begin{tabular}{|c|c|c|c|}
\hline Equilibrium point & $\operatorname{Det}(\mathrm{J})$ Symbol & $\operatorname{Tr}(\mathrm{J})$ Symbol & Result \\
\hline $\mathrm{O}: \mathrm{x}=0, \mathrm{y}=0$ & + & - & ESS \\
\hline $\mathrm{W}: \mathrm{x}=0, \mathrm{y}=1$ & + & + & Unstable \\
\hline $\mathrm{U}: \mathrm{x}=1, \mathrm{y}=0$ & + & + & Unstable \\
\hline $\mathrm{V}: \mathrm{x}=1, \mathrm{y}=1$ & + & - & ESS \\
\hline$s^{*}: x^{*}=\frac{2(\Delta i-\Delta r)}{i+2 \Delta i}, y^{*}=\frac{2(\Delta i-\Delta r)}{i+2 \Delta i}$ & - & 0 & Saddle Point \\
\hline
\end{tabular}

Table 2 shows that $\mathrm{O}(0,0)$ and $\mathrm{V}(1,1)$ of the five equilibrium points is the evolutionary stable point. $\mathrm{W}(0,1)$ and $\mathrm{U}(1,0)$ is instability point, $\mathrm{S}(\mathrm{x}, \mathrm{y})$ is saddle point. Evolution stable point $\mathrm{O}(0,0)$ show that Under the cooperation strategy of enterprise $A$ and $B$, If one of the companies do not chooses to participate in the research and development of the alliance, B will also choose not to participate. At this point, the enterprise game result is the ESS point $O(0,0)$. That is [not participating, not participating], But once an enterprise breaks the steady state equilibrium, choose the participation strategy. For another company. under the premise of cooperation constraint, we can jointly undertake the new cost of $\mathrm{r} \& \mathrm{~d}$. Therefore, I also choose to participate in the strategy. The game result is the ESS point V $(1,1)$, [participation, participation] strategy.

A two-dimensional phase diagram is used to describe the dynamic evolution trajectory under table 2. As shown in Figure 1 .

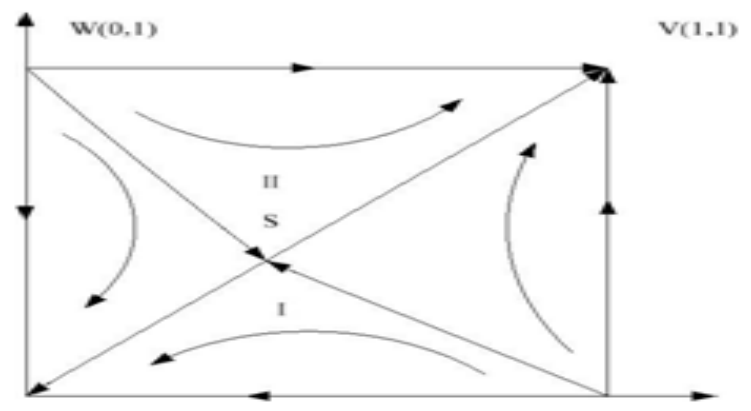

Fig.1 System dynamic evolution diagram

The dynamic process of enterprise cooperation technology innovation game can be analyzed from figure 1. The line W-S-U of two unstable points $\mathrm{W}$ and $\mathrm{U}$ and saddle point $\mathrm{S}$ is the boundary of different states. The initial state is in the upper right region of the fold line SII (WSUVw), the system will converge to the ESS point $\mathrm{V}(1,1)$, the initial state is in the lower left area of the fold line $\mathrm{S}$ (wsUOw), the system will converge to the ESS point $\mathrm{O}(0,0)$. The result of long-term evolutionary game between enterprise A and enterprise B is to realize the steady state equilibrium of [participation, participation] or [non-participation, non-participation]. It depends on the size and the area in figure 1.If $s_{1}=s_{11}$, the probability of using [participation, participation] is the same as the probability of not participating and not participating. If $s_{1}<s_{11}$, the probability of the system using [not participating, not participating] is greater than the probability of [participation, participation].On the contrary, it tends to achieve [participation, participation] balance.

Further, because of $s_{1}=\frac{2(\Delta i-\Delta r)}{i+2 \Delta i}$, so $\frac{\partial s_{1}}{\partial \Delta r}=-2 \frac{1}{i+2 \Delta i}$ by $i+2 \Delta i>0$ get $\frac{\partial s_{1}}{\partial \Delta r}<0 \quad s_{1}$ is the monotone decreasing function of $\Delta r$, with the new revenue increasing of the participation of the alliance, area is reducing,this is saddle point. The probability of enterprise selection [participation and participation] is gradually increasing. Achieving a stable equilibrium ESS point V(1,1), when $\Delta r$ is decreaseing, the are of $s_{1}$ is increasing, the probability of converging to the ESS point $\mathrm{O}(0,0)$ increases. 
It means that the stronger the innovation incentive effect, the higher the expected gain of $\mathrm{r} \& \mathrm{~d}$ cooperation and the higher the probability level of cooperative participation. On the other hand, in consideration of $\frac{\partial s_{1}}{\partial \Delta i}=2 \frac{i+2 \Delta r}{(i+2 \Delta i)^{2}}>0$ and $\frac{\partial s_{1}}{\partial i}=-2 \frac{\Delta i-\Delta r}{(i+2 \Delta i)^{2}}<0$

$0<s_{1}<1$ and $i+2 \Delta i>0$ inference $-\frac{1}{2} i<\Delta r<\Delta i$ at this moment, $s_{1}$ is new cost of $\mathrm{r} \& \mathrm{~d}$ cooperation. $\Delta i$ is also the monotone increasing function of total $\mathrm{r} \& \mathrm{~d}$ cost $i$.The increase or decrease of $\Delta i$ and $i$ will cause result of the game to converge steady in two different directions: the ESS point $\mathrm{O}(0,0)$ or $\mathrm{V}(1,1)$.

That is to say, on the control of enterprise cooperation, whether to participate in the production in the process of enterprise $r \& d$ alliance cooperation innovation platform, depends on the gain $\Delta r$, cooperation $\Delta i$, cooperation between production cost and the size of the total development cost $i$. While $\Delta r>\Delta i$, the enterprise will converge to the ESS point $\mathrm{V}(1,1)$. At this time, the enterprise decision is [participation, participation], While $\Delta r<\Delta i$, The game result of the enterprise will converge to the ESS point $\mathrm{O}(0,0)$. At this time, the enterprise decision is [not participating, not participating]. The greater the cooperative $\mathrm{r} \& \mathrm{~d}$ gain $\Delta r$, the higher the probability of the participation of enterprises in cooperative research and development. However, if the new cost increases, the willingness to participate will be reduced. Considering the above analysis is in a neglected under the premise of government intervention, which is high for some initial research and development costs, cooperative r\&d capital intensive new technology project, if it can be based on government subsidies tools to ensure that the co-operative research and development, will greatly improve the enterprise's desire to participate in the alliance cooperation between production research and development, at the same time, which further confirmed in production union government subsidies to accelerate technology transfer to promote collaborative innovation incentive effect.

\section{3. game analysis of enterprise r\&d decision-making under the strategy of non-cooperation among enterprises.}

The above analysis is based on enterprise $\mathrm{A}$ and $\mathrm{B}$, once participating in the production and learning alliance, then the company will carry out research and development among enterprises, share the r\&d cost, and share the innovation benefits.But as Serone puts it, in a large number of internal production alliance, even expected to enterprise cluster cooperation overall higher returns, also tend to choose competitive strategies rather than cooperation, forming a co-operative research within the prisoner's dilemma.The following is based on non-cooperative (competitive) strategic premise analysis ${ }^{[11]}$

If the strategy probability of enterprise $\mathrm{A}$ is still $\mathrm{x}$, the probability of the corresponding selection of enterprise $\mathrm{B}$ is, and the probability of $\mathrm{H} 1$ and $\mathrm{H} 3$ is satisfied. The r\&d income matrix of enterprise A and B is shown in table 3.

Tab. 3 payoff matrix of cooperative innovation

\begin{tabular}{|c|c|c|}
\hline & Participate & Does not participate \\
\hline Participate & $\frac{1}{2} r-i-\Delta i, \frac{1}{2} r-i-\Delta i$ & $\frac{1}{2} r-i+\Delta r-\Delta i, \frac{1}{2} r-\Delta r-i$ \\
\hline $\begin{array}{l}\text { Does not } \\
\text { participate }\end{array}$ & $\frac{1}{2} r-\Delta r-i, \frac{1}{2} r-i+\Delta r-\Delta i$ & $\frac{1}{2} r-i, \quad \frac{1}{2} r-i$ \\
\hline
\end{tabular}

At this point, the income function of enterprise A can be calculated as

$$
\begin{aligned}
& u_{1 y}=\left(\frac{1}{2} \mathrm{r}-i-\Delta i\right) y+\left(\frac{1}{2} \mathrm{r}-i+\Delta r-\Delta i\right)(1-y) \quad u_{1 n}=\left(\frac{1}{2} \mathrm{r}-i-\Delta r\right) y+\left(\frac{1}{2} \mathrm{r}-i\right)(1-y) \\
& \bar{u}_{1}=u_{1 y} x+u_{1 n}(1-x) \quad \text { There are } \quad u_{1 y}-\bar{u}_{1}=x(1-x)(\Delta r-\Delta i)
\end{aligned}
$$


Therefore, the replication dynamic equation of enterprise A is:

$$
\frac{d x}{d t}=x\left(u_{1 y}-\bar{u}_{1}\right)=x(1-x)(\Delta r-\Delta i)
$$

Similarly, for enterprise $\mathrm{B}$, the expected return is.

$$
\begin{aligned}
& u_{2 y}=\left(\frac{1}{2} \mathrm{r}-i-\Delta i\right) x+\left(\frac{1}{2} \mathrm{r}-i+\Delta r-\Delta i\right)(1-x) \quad u_{2 n}=\left(\frac{1}{2} \mathrm{r}-i-\Delta r\right) x+\left(\frac{1}{2} \mathrm{r}+\Delta r-i-\Delta i\right)(1-x) \\
& \bar{u}_{2}=u_{2 y} y+u_{1 n}(1-y) \quad \text { Therefore } \quad u_{2 y}-\bar{u}_{2}=(1-y)(\Delta r-\Delta i)
\end{aligned}
$$

Therefore, the dynamic equation of $\mathrm{B}$ enterprise replication is $\frac{d y}{d t}=y\left(u_{2 y}-\bar{u}_{2}\right)=y(1-y)(\Delta r-\Delta i)$, let $\frac{d x}{d t}=\frac{d y}{d t}=0$

In the plane of $M=\{(x, y) \mid 0 \leq x \leq 1,0 \leq y \leq 1\}$, We can obtain the four equilibrium points of this evolutionary game.They are respectively: $(0,0),(0,1),(1,0),(1,1)$

$$
\text { Similarly, the jacobian matrix is }\left[\begin{array}{ll}
(1-2 x)(\Delta r-\Delta i) & 0 \\
0 & (1-2 y)(\Delta r-\Delta i)
\end{array}\right]
$$

The determinant and trace of the matrix are shown in table 4.

According to the above equation, the game income level of enterprise A and B is highly dependent on the symbol.The following will do evolutionary analysis for $\Delta r-\Delta i>0$ and

\begin{tabular}{|c|c|c|c|}
\hline Equilibrium point & $\operatorname{Det}(J)$ Symbol & $\operatorname{Tr}(\mathrm{J})$ Symbol & Result \\
\hline $\mathrm{O}: \mathrm{x}=0, \mathrm{y}=0$ & + & + & Unstable \\
\hline $\mathrm{W}: \mathrm{x}=0, \mathrm{y}=1$ & - & 0 & Saddle Point \\
\hline $\mathrm{U}: \mathrm{x}=1, \mathrm{y}=0$ & - & 0 & Saddle Point \\
\hline $\mathrm{V}: \mathrm{x}=1, \mathrm{y}=1$ & + & - & ESS \\
\hline
\end{tabular}
$\Delta r-\Delta i<0$.First, the evolution process of game strategy under the constraints of $\Delta r-\Delta i>0$

is given (see table 4 )

Tab. 4 the result of evolutionary stability

Table 4 shows that $\mathrm{V}(1,1)$ in the 4 equilibrium points is an evolutionary stable point. $\mathrm{O}(0,0)$ is unstable, $\mathrm{W}(0,1)$ and $\mathrm{U}(1,0)$ are saddle points (as shown in figure 2). While $\Delta r-\Delta i>0$, constraints in production alliance cooperation strategy, enterprises choose competitive engagement strategies to obtain additional earnings, but if you choose not to participate in, means that the enterprise overall revenue absolute decrease, so the game is based on self-interest maximization goal, will choose [involvement, participation in] a consistent strategy, eventually the two sides game result of convergence to the ESS point $\mathrm{V}(1,1)$.

A two-dimensional phase diagram is used to describe the dynamic evolution trajectory under

\begin{tabular}{|c|c|c|c|}
\hline Equilibrium point & $\operatorname{Det}(J)$ Symbol & $\operatorname{Tr}(\mathrm{J})$ Symbol & Result \\
\hline $\mathrm{O}: \mathrm{x}=0, \mathrm{y}=0$ & + & - & ESS \\
\hline $\mathrm{W}: \mathrm{x}=0, \mathrm{y}=1$ & - & 0 & Saddle Point \\
\hline $\mathrm{U}: \mathrm{x}=1, \mathrm{y}=0$ & - & 0 & Saddle Point \\
\hline $\mathrm{V}: \mathrm{x}=1, \mathrm{y}=1$ & + & + & Unstable \\
\hline
\end{tabular}
table 4.

While $\Delta r-\Delta i<0$, The sign of determinant and trace is shown in table 5.

Tab. 5 the result of evolutionary stability

Table 5 shows that $\mathrm{O}(0,0)$ in the four equilibrium points is the evolutionary stable point, $\mathrm{W}(0,1)$, $\mathrm{U}(1,0)$ and $\mathrm{V}(1,1)$ are unstable points. In fact, under the constraint of non-cooperative strategy, the 
enterprises participating in the production learning alliance should bear the burden of independent research and development of new costs $\Delta i$, is greater than cooperative gain.Therefore, enterprises must choose not to participate in the strategy. At this point, the game converges to the problem ESS point $\mathrm{O}(0,0)$, and the enterprise strategy is [not participating, not participating].

A two-dimensional phase diagram is used to describe the dynamic evolution trajectory under table 5.

Considering the property right definition and protection of the research and development between enterprises in the Chinese market, there is not enough legal guarantee, which leads to the high cost of recourse. If competitive production enterprises for non cooperative alliance, so in some innovative technologies long return period, huge initial $r \& d$ projects, technology transfer, rather than cooperation may be a more appropriate, can accelerate the alliance between production technology and knowledge transfer mode of cooperation, In the transfer mode, the technology owner (learning) can transfer technology to enterprise A and B simultaneously. Assuming that the benefits of the technology transfer are unchanged, It can make the new cost of the enterprise under the cooperative research and development of the manufacturing alliance become $\Delta i$ to $\Delta i / 2$, so as to guarantee $\Delta r-\Delta i / 2>0$. Further, by strengthening the enterprise income, the game strategy of the enterprise is transformed from [not participating, not participating] to non-cooperative constraint [participation,participation].

\section{Simulation analysis of enterprise $r \& d$ evolution game.}

In order to further describe the gain in the different initial probability level and the research and development of production $\Delta r$. The new cost $\Delta i$ and the change of $r \& d$ input cost $i$ will influence the selection of cooperation between enterprises and non-cooperation, participation and non-participation strategies.Based on the two conditions of cooperation and non-cooperation, this paper carries out the numerical simulation of the dynamic equation of the enterprise income function respectively to reveal the influence of the decision-making process and control parameters in the research and development of the enterprise.

\section{1game simulation analysis under the premise of cooperation strategy.}

\subsubsection{The influence of enterprise game strateg}

The participation probability of enterprise $\mathrm{A}$ and $\mathrm{B}$ is set as $x_{0}=y_{0}=0.3$, take $\Delta r$ gradient to the interval $[0.3,0.7]$, The simulation results of the income dynamic equation are shown in figure 2.

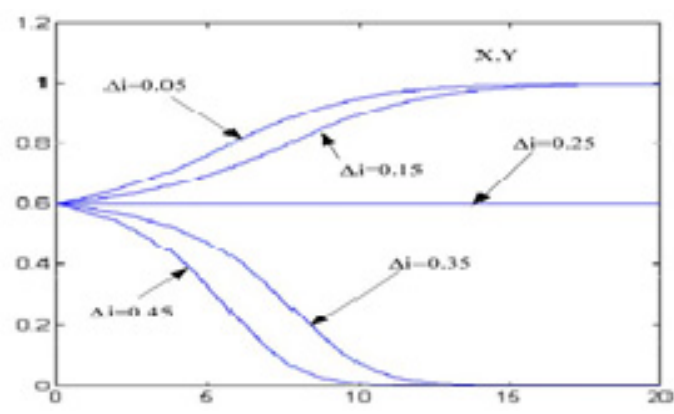

Fig. 2 Simulation of the influence of $\Delta r$ on the evolutionary results

While Copying the dynamic system initial point value $x_{0}=y_{0}=0.3$, when the initial point value of the replication dynamic system is $x_{0}=y_{0}=0.3$, When the gain is $\Delta r=0.3$ for the production study cooperation, the two parties are at the boundary of participation and non-participation strategy. It's in a straight line. 
While $\Delta r>0.3$, the profit of the game based on the cooperative research and development of the production alliance gains amplification, the probability of an enterprise choosing to participate in the strategy has also increased. At the same time, this participation decision has the following effect, The participation of one enterprise will lead to the transformation of another enterprise into the participation decision to share the benefits. At this time, the two sides of the game tend to be the ESS point $\mathrm{V}(1,1)$, namely [participation, participation] strategy.

On the contrary, while $\Delta r<0.3$, he game players gain a small amount of cooperation, and if you consider the additional cost $\Delta i$ of participating in the production learning alliance. The game players will abandon their participation strategy and turn to non-participating strategies. In the same way, the other side of the game will also choose not to participate in the strategy, and then the two sides tend to be the ESS point $\mathrm{O}(0,0)$. The simulation results further confirm that the cooperative innovation of enterprises participating in the production learning alliance is directly related to the cooperative gain level $\Delta r$ of cooperative $r \& d$. So the current our country of the government's focus on common alliance between production in enterprise is direct subsidy policy has its rationality, this policy can strengthen the enterprises to participate in learning alliance the innovation incentives.

\subsubsection{The influence of $\Delta i$ on enterprise game strategy}

Under constraint of $-\frac{1}{2} i<\Delta r<\Delta i$,order $i=2, \Delta r=0.5$, take the gradient in the interval [0.05,0.45]

5. The simulation results of the income dynamic equation are shown in figure 3.

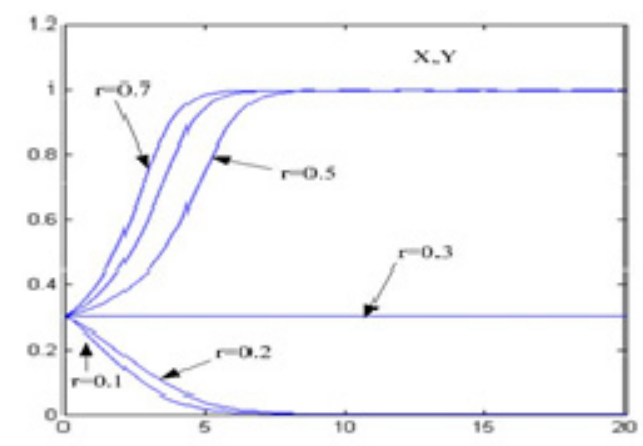

Fig.3 Simulation of the influence of $\mathbf{i}$ on the evolutionary results

When the cooperation input is $\Delta i=0.25$, he two sides of the game are engaged and not participating in the strategic transformation. While $\Delta i>0.25$, the net income $\Delta r-\Delta i$ of cooperation between the players participating in the production and learning alliance is reduced. At this point, the enterprise is inclined to be converted from participating strategies to not participating. The strategy identity is based on the steady-state ESS point, and the other player will choose not to participate in the strategy. At this point, the two sides of the game tend to the ESS point $\mathrm{O}(0,0)$, i.e., no participation, no participation. While $\Delta i<0.25$, the new cost $\Delta i$ of cooperation is reduced, which leads to the increase of net income of cooperation. Therefore, the game party tends to participate in the strategy. Similarly, the other player will choose to participate in the strategy.At this time, the game players tend to be open and open.

According to the comprehensive view, the simulation results further confirm that the cooperative innovation of enterprises participating in the production and learning alliance is directly related to the cooperative gain level $\Delta r$ of cooperative $\mathrm{r} \& \mathrm{~d}$ and the cooperation $\mathrm{r} \& \mathrm{~d}$ cost $\Delta i$. Considering the production knowledge and technical advantages in the alliance party (learn) often have is dominant, and therefore the current our country of the government's focus on common alliance between production in enterprise is direct subsidy policy has its rationality, This policy can strengthen the incentive of enterprise participation by directly increasing the income level of enterprises participating in the collaborative innovation of production. But due to the condition of $\Delta r<\Delta i$, no matter how $\mathrm{r} \& \mathrm{~d}$ costs change, enterprise have convergence in ESS points $\mathrm{O}(0,0)$, is not involved in strategy, thus the actual incentive effects of government subsidies still depends on 
the intensity of government subsidies. The incentive effect can only be revealed if the profit and loss conditions for the cooperative innovation of the production study alliance are changed from $\mathrm{x}$ to $\mathrm{y}$ through subsidies.

\section{2 game simulation analysis under the premise of non-cooperative strategy}

Firstly, analyze the change of enterprise game strategy under constraint of $\Delta r<\Delta i$.Take the initial point value changed to the interval $[0.1,0.6]$, and take the total $\mathrm{r} \& \mathrm{~d} \operatorname{cost} i=2$. And ave the figure 4.



Fig.4 Simulation diagram at $\Delta r<\Delta i$

According to the figure 4, under constraints of $\Delta r<\Delta i$, due to the premise of non-cooperative strategy, the net income change $\Delta r-\Delta i$ of technological innovation of enterprises choosing to participate in the production learning alliance is negative. Therefore, regardless of the initial participation probability level of the enterprise, the game result is to converge to the steady-state ESS point $\mathrm{O}(0,0)$, namely [not participating, not participating] strategy. Further,while $\Delta r>\Delta i$, take the initial point value gradually to the interval $[0.1,0.6]$, and take the total $\mathrm{r} \& \mathrm{~d} \operatorname{cost} i=2$, have the figure 5.

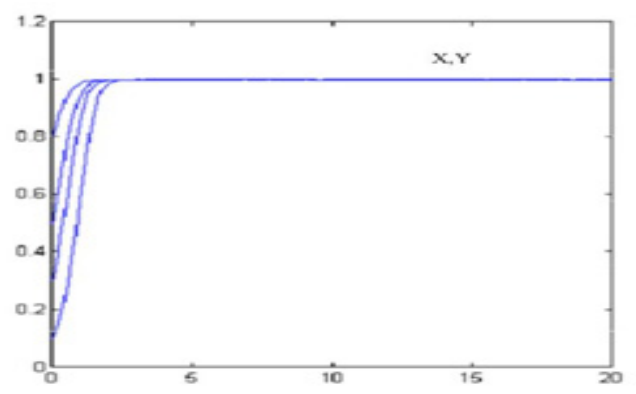

Fig.5 Simulation diagram at $\Delta r>\Delta i$

Under non-cooperative constraints, it this time, the net income level of cooperative research and development is positive. Regardless of the initial probability level, the results of the final game of the enterprise converge to the ESS point V $(1,1)$. At this time, the enterprise decision is to participate in the production and learning collaborative innovation.

\section{Reference:}

[1] Hurry K, Salter A. Open for Innovation: the Role of Openness in Explaining Innovation Performance among U.K Manufacturing Firms[J]. Strategic Management Journal, 2003, 27(2): 131-150.

[2] Groger .M. The Competitive Advantage of Nat ions[M]. New York: The Free Press, 2008.

[3] Hitt M A, Hoskisson R E, Kim H. International Diversification: Effects on Innovation and Firm Performance in Product-Diversified Firms[J]. Academy of Management Journal, 1997, 40(4):767-798. 
[4] Lichtenthaler U. Open Innovation in Practice: An Analysis of Strategic Approaches to Technology Transactions[J]. IEEE Transactions on Engineering Management, 2008, 55(1):148-157.

[5] Suxianna,Xiefuji. The evolution game analysis of enterprise innovation strategy selection [J]. Research and development management, 2016(2):12-20

[6] Fisher Carbone, Jesús Contreras, Josefa Z. Hernández, et al. Open Innovation in an Enterprise 3.0 framework: Three case studies [J]. Expert Systems with Applications,2012, 39(10): 8929-8939.

[7] Leijing,Panjieyi. Game analysis of strategic mode selection of enterprise cooperation innovation [J], intelligence magazine. 2009 (28) 5:18-25

[8] Nelson W. Organizing for Open Innovation: Focus on the Integration of Knowledge [J]. Organizational Dynamics, 2010, 39 (2): 145-154

[9] Hejunli, The choice of cooperative strategy among enterprises in the production alliance -based on the analysis of inter-temporal non-cooperative game $[\mathrm{J}]$, journal of shanxi university of finance and economics. 2015, 4:78-87

[10]Gann D M. H. Chesbrough, Open Innovation: The New Imperative For Creating And Profiting From Technology, Harvard Business School Press, 2003 (272 pp. \$35.00, ISBN: 1-57851-837-7).[J]. Research Policy, 2005, 34(1):122-123.

[11]Janeiro P, Proença I, Gonçalves V D C. Open innovation: Factors explaining universities as service firm innovation sources $\succsim$ [J]. Journal of Business Research, 2013, 66(10):2017-2023. 